\title{
ATUAÇÃO DO ENFERMEIRO NO CONTEXTO DA VIOLÊNCIA CONTRA A MULHER: REVISÃO INTEGRATIVA DA LITERATURA
}

\author{
NURSE'S PERFORMANCE IN THE CONTEXT OF VIOLENCE AGAINST WOMEN: AN INTEGRATIVE \\ LITERATURE REVIEW
}

\section{RENDIMIENTO DE LA ENFERMERA EN EL CONTEXTO DE LA VIOLENCIA CONTRA LAS MUJERES: UNA REVISIÓN INTEGRATIVA DE LA LITERATURA}

\author{
Fabiana dos Santos Alecrim¹, Janaina Regina Augusto², Sheyla Cristina de Oliveira ${ }^{3}$, \\ Suelen Rodrigues de Santana ${ }^{4}$, Meline Rossetto Kron Rodrigues ${ }^{5}$
}

Submetido: $15 / 02 / 2020$

Aprovado: 26/06/2020

\begin{abstract}
RESUMO
Objetivo: identificar e analisar o papel do enfermeiro no contexto da violência contra a mulher. Método: revisão integrativa realizada nas bases de dados LILACS, BDENF-Enfermagem, MEDLINE, IBECS-ES e Coleciona SUS. Os descritores em saúde (DeCS) foram: "Violência contra a Mulher" e "Enfermeiras e Enfermeiros". Foram selecionados os artigos indexados nestas bases que tiveram de acordo com a temática. Foram excluídos estudos de revisão integrativa e de literatura, livros, capítulos e resenhas de livros, manuais, relatórios técnicos. Artigos que não possuíam relação com a questão norteadora também foram excluídos. Resultados: Foram incluídos cinco estudos que evidenciam que enfermeiros no contexto da violência contra a mulher buscam prestar atendimento integral às vítimas para que haja recuperação da saúde física, permeada pela expectativa de compreender a situação buscando proporcionar bem-estar emocional, apoio e a continuidade do cuidado. Estabelecer vínculo com as pacientes é necessário para o cuidado, bem como o trabalho multiprofissional neste contexto.
\end{abstract}

DESCRITORES: Enfermeiras e Enfermeiros; Violência Doméstica; Violência contra a Mulher.

\section{ABSTRACT}

Objective: to identify and analyze the role of nurses in the context of violence against women. Method: an integrative review carried out in the LILACS, BDENF-Enfermagem, MEDLINE, IBECS-ES and Coleciona SUS databases. The health descriptors (DeCS) were: "Violence against Women" and "Nurses". Articles indexed in these databases were selected according to the theme. Integrative and literature review studies, books, chapters and book reviews, manuals, technical reports were excluded. Articles that were not related to the guiding question were also excluded. Results: Five studies were included that show that nurses in the context of violence against women seek to provide comprehensive care to victims so that there is recovery of physical health, permeated by the expectation of understanding the situation seeking to provide emotional well-being, support and continuity of care. caution. Establishing a bond with patients is necessary for care, as well as multiprofessional work in this context.

KEYWORDS: Nurses; Domestic Violence; Violence Against Women

1 Discente de Enfermagem da Universidade UNG, Guarulhos, São Paulo. https://orcid.org/0000-0002-1325-6326, e-mail: fabiana_alecrim@hotmail.com

2 Discente de Enfermagem da Universidade UNG, Guarulhos, São Paulo https://orcid.org/0000-0003-0678-1496, e-mail: janaregina16@gmail.com

3 Discente de Enfermagem da Universidade UNG, Guarulhos, São Paulo https://orcid.org/0000-0002-0799-7507, e-mail: sheyla_cristi@hotmail.com

4 Discente de Enfermagem da Universidade UNG, Guarulhos, São Paulo https://orcid.org/0000-0002-9831-1469, e-mail: suelensantana60298@gmail.com

5 Enfermeira. Professora Doutora, do Programa de Pós-graduação Stricto Sensu em Enfermagem da Universidade UNG, Guarulhos, SP, Brasil. https://orcid.org/0000-0003-2174-268X, e-mail: me_kron@hotmail.com 


\section{RESUMEN}

Objetivo: identificar y analizar el papel de las enfermeras en el contexto de la violencia contra la mujer. Método: revisión integradora realizada en las bases de datos LILACS, BDENF-Enfermagem, MEDLINE, IBECS-ES y Coleciona SUS. Los descriptores de salud (DeCS) fueron: "Violencia contra la mujer" y "Enfermeras y enfermeras". Los artículos indexados en estas bases de datos fueron seleccionados de acuerdo con el tema. Se excluyeron los estudios integrales y de revisión de literatura, libros, capítulos y reseñas de libros, manuales, informes técnicos. También se excluyeron los artículos que no estaban relacionados con la pregunta guía. Resultados: se incluyeron cinco estudios que muestran que las enfermeras en el contexto de la violencia contra las mujeres buscan brindar atención integral a las víctimas para que se recupere la salud física, impregnada por la expectativa de comprender la situación que busca proporcionar bienestar emocional, apoyo y continuidad de la atención. precaución. Establecer un vínculo con los pacientes es necesario para la atención, así como el trabajo multiprofesional en este contexto.

DESCRIPTORES: Enfermeras y Enfermeros; Violencia Doméstica; Violencia contra la Mujer 


\section{INTRODUÇÃO}

A violência doméstica se trata de um problema mundial, de origem indefinível e multifatorial, que na maioria das vezes tem como alvo vítimas do sexo feminino. Esse contexto apresenta forte relação com conceitos sociais e culturais estabelecidos anteriormente nas relações de gênero e quanto à visão do papel da mulher na sociedade de maneira geral ${ }^{1}$.

O mapa da violência contra a mulher revela que a cada 15 segundos, uma mulher é agredida no Brasil e a cada duas horas uma é assassinada. Segundo registros realizados pela ONU (Organização das Nações Unidas), o Brasil é o $5^{\circ}$ colocado entre 83 países no ranking de homicídios de mulheres. Esse levantamento possibilita identificar o problema como uma questão de saúde pública, que exige o avanço de políticas voltadas ao cuidado integral à mulher e preparo adequado dos profissionais responsáveis pelo acolhimento e acompanhamento de vítimas nessa situação².

A violência doméstica e familiar contra a mulher é definida pela Lei 11.340/06, intitulada lei Maria da Penha, como qualquer "ação ou omissão baseada no gênero que the cause morte, lesão, sofrimento físico, sexual ou psicológico e dano moral ou patrimonial". Essa lei garante que estados e municípios assegurem os direitos à saúde da mulher, bem como organizam e implantam redes de apoio e programas de saúde que a atendam integralmente as mulheres vítimas de violência, além de visar diminuir os índices de delitos cometidos contra as mesmas $^{3}$.

A violência doméstica é fator de adoecimento não somente da vítima, mas também de toda família, pois consequentemente acarreta danos biopsicossociais e posteriormente alta demanda no serviço de saúde pública².

A mulher por ser alvo preferencial desse tipo de violência, tem merecido a atenção por parte de profissionais, principalmente os de enfermagem que na sua trajetória prática e em qualquer ambiente de trabalho pode defrontar-se com essa situação, exigindo conhecimento específico e habilidade para realizar esse cuidar como expressão humanizadora da enfermagem, com poder transformador, que deve ser sentido e vivido por parte de quem cuida e de quem é cuidado1.

Nessa concepção, o primeiro contato da mulher no serviço de saúde deve acontecer com os profissionais de enfermagem, que farão o acolhimento humanizado a realização da anamnese, coleta de material para exames laboratoriais, agendamento de retorno e administração de medicações. Esses são passos que garantem a aderência e seguimento ao tratamento ${ }^{4}$.

A partir disso é possível compreender o destaque do papel do enfermeiro como protagonista, líder da equipe de enfermagem e a frente ao contato inicial e acolhimento na atenção primária, sendo considerada referência no acompanhamento familiar através da Estratégia de Saúde da Família (ESF). A atenção primária possibilita um olhar ampliado baseado na realidade e demanda de cada família pertencente ao território, permitindo a atuação do mesmo desde ações preventivas, até todos os procedimentos necessários quando constatada a violência de fato ${ }^{4-5}$.

Tendo conhecimento dessa realidade, é de suma importância que o enfermeiro e sua equipe reconheçam seu papel diante de tal situação e busquem atuação efetiva, considerando a violência doméstica como algo relevante e não permitindo que seja considerado como algo comum, mas como uma questão que precisa ser solucionada ofertando alternativas aos que necessitam de ajuda ${ }^{5}$.

Além do conhecimento científico que permite atuação em todas as etapas, é importante envolver toda a família neste contexto, além de contar com a atuação multidisciplinar e buscar recursos e serviços intersetoriais, para que cada setor atue de maneira efetiva e resolutiva, visando prover mudança na realidade da muIher atendida ${ }^{5-6}$.

Faz parte do contexto da enfermagem, bem como do enfermeiro, acompanhar a trajetória da vítima e promover o fortalecimento do vínculo já existente, estabelecendo uma relação de confiança, e deste modo, fazer com que a vítima se sinta acolhida e protegida.

Diante do exposto, percebe-se a atuação do enfermeiro e sua equipe como parte fundamental no que se refere ao processo de apoio às vítimas de violência doméstica, possibilitando a ampliação do olhar da vítima mediante sua situação, e seu acesso a alternativas desconhecidas anteriormente, além de proporcionar recursos para a superação deste ciclo vicioso ${ }^{6}$. O objetivo do presente trabalho é identificar e analisar o papel do enfermeiro no contexto da violência doméstica contra a mulher.

\section{MÉTODO}

Foi utilizado o método de revisão integrativa da literatura, que busca constatar e sumarizar qual a produção científica disponível acerca da temática em questão, 
com a finalidade de conhecer o que se sabe sobre o assunto e subsidiar novos estudos ${ }^{7}$.

Para elaboração da revisão foram concretizadas seis etapas: na primeira etapa foi definida as questões principais da pesquisa; na segunda etapa foram definidos os critérios de inclusão e exclusão; na terceira etapa foram selecionadas as bases de dados e realizado a busca dos artigos científicos; na quarta etapa foi realizado a análise dos dados; na quinta etapa foi realizado a discussão dos achados e finalmente na sexta etapa a síntese da revisão foi apresentada.

A pergunta norteadora desta revisão foi: Qual o papel do enfermeiro no contexto da violência doméstica feminina? Para realizar as buscas nas bases de dados, foram utilizados os DeCS (Descritores em Ciências da Saúde) e seus respectivos sinônimos de "Violência contra a Mulher" e "Enfermeiras e Enfermeiros" conectados pelo operador boleando "and". O acesso às bases de dados virtuais ocorreu no mês de maio do ano de 2019.

A triagem dos artigos elegíveis foi realizada por dois revisores, buscando garantir rigor metodológico na seleção dos artigos nas bases de dados. As bases eletrônicas consultadas foram: Literatura Latino-Americana e do Caribe em Ciências da Saúde (LILACS), Literatura Internacional em Ciências da Saúde (MEDLINE) e BDENF - Enfermagem no sítio da Biblioteca Virtual em Saúde (BVS).

Foram utilizados estudos publicados e indexados na base de dados acima referidos. Os critérios de inclusão serão: artigos publicados acerca da temática e publicados até a data da busca. Não houve restrição de idioma.

Os critérios de exclusão foram: revisão integrativa e de literatura, livros, capítulos e resenhas de livros, manuais, relatórios técnicos. Também foram excluídos artigos que não possuíam relação com a questão norteadora do estudo.

A triagem e seleção de artigos foram apresentadas por diagrama de fluxo de estudos e posteriormente houve extração dos conceitos abordados em cada artigo e os trabalhos de acordo com seu conteúdo. Os resultados foram apresentados em forma de tabela e discutidos com os achados da literatura.

\section{Aspectos éticos}

Por tratar-se de estudo secundário da literatura, não foi necessário a aprovação do Comitê de Ética em Pesquisa (CEP).

\section{RESULTADOS}

Nas buscas nas bases de dados, foram resgatados 56 artigos, sendo 24 na BDENF - Enfermagem, 24 no LILACS, 01 no MEDLINE, 6 no BECS e 1 no Coleciona SUS. Inicialmente foi realizada a triagem por título, e 22 artigos foram excluídos nesta etapa. Sequencialmente a triagem seguiu pela leitura dos resumos e 12 artigos foram excluídos. Finalmente cinco artigos foram incluídos para análise, conforme expressa o diagrama de fluxo de estudos selecionados. Posteriormente foram extraídos os conceitos abordados em cada artigo e os trabalhos foram descritos conforme seu conteúdo.

Figura 1. Diagrama de fluxo dos estudos selecionados para elaboração de revisão integrativa da literatura para análise dos cuidados de enfermagem prestados às vítimas de violência doméstica.

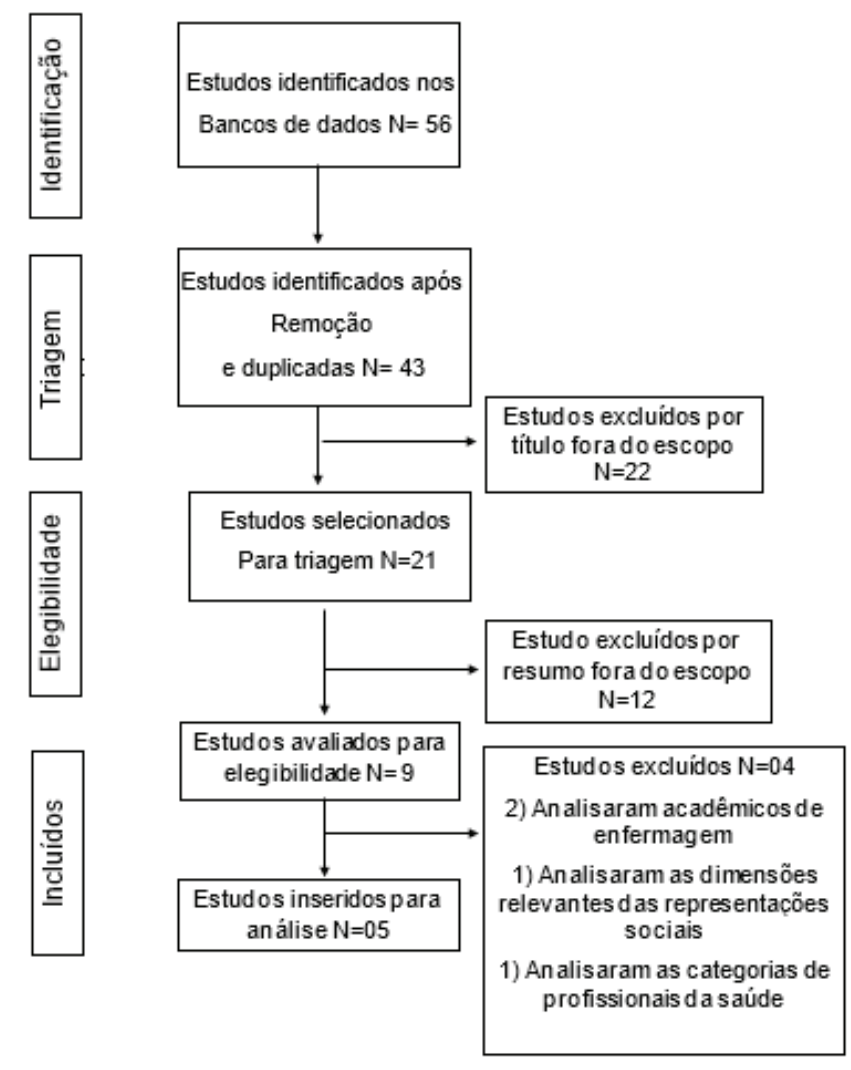

Elaboração: Os autores, 2019

Nesta revisão integrativa da literatura, foram inseridos para análise cinco artigos publicados no período de 2014 a 2018, referentes ao papel do enfermeiro no contexto da violência doméstica contra a mulher.

Os artigos analisados que preencheram os cri- 
térios de inclusão previamente estabelecidos, estão sumarizados na Tabela 1, segundo autoria, título do artigo, revista e ano de publicação. A tabela 2 sumariza os artigos inseridos segundo seus objetivos, instrumento utilizado e síntese dos resultados e conclusões dos estudos analisados.

Tabela 1- Caracterização dos estudos incluídos na análise:

\begin{tabular}{|c|l|l|l|}
\hline \multicolumn{1}{|c|}{ Autores } & \multicolumn{1}{|c|}{ Título do Artigo } & Revista/Ano da Publicação \\
\hline 01 & $\begin{array}{l}\text { Bruna Lais Alcará de Morais; Maria Aux- } \\
\text { iliadora de Souza Gerk; Cristina Brandt } \\
\text { Nunes }^{8}\end{array}$ & $\begin{array}{l}\text { Enfermeira da Estratégia de Saúde da } \\
\text { Família: abordagem frente à mulher em } \\
\text { situação de violência }\end{array}$ & Nursing (São Paulo) 2018 \\
$\begin{array}{l}\text { Leônidas de Albuquerque Netto; Eric Rosa } \\
\text { Tereira; Joyce Martins Arimatea Branco } \\
\text { Priscilla Valladares Broca9 }\end{array}$ & $\begin{array}{l}\text { Atuação da enfermagem na conservação } \\
\text { da saúde de mulheres em situação de } \\
\text { violência }\end{array}$ & REME rev. min. enferm (2018) \\
\hline 03 & $\begin{array}{l}\text { Laura Ferreira Cortes; Stela Maris de Mello } \\
\text { Padoin }{ }^{10}\end{array}$ & $\begin{array}{l}\text { Intencionalidade da ação de Cuidar } \\
\text { mulheres em situação de violência: con- } \\
\text { tribuições para a Enfermagem e Saúde }\end{array}$ & $\begin{array}{l}\text { Esc. Anna Nery Rev. Enferm } \\
\text { (2016) }\end{array}$ \\
\hline 04 & $\begin{array}{l}\text { Rosilene Santos Baptista; Olga Benário } \\
\text { Batista de Melo Chaves; Inacia Sátiro } \\
\text { Xavier de França; Francisco Stélio de } \\
\text { Sousa; Michelly Guedes de Oliveira; Carla } \\
\text { Carolina da Silva Leite }\end{array}$ & $\begin{array}{l}\text { Violência sexual contra mulheres: a prática } \\
\text { de enfermeiros }\end{array}$ & Rev. RENE (2015) \\
\hline 05 & $\begin{array}{l}\text { Joselito Santos; Roberta Leal de Andrade; } \\
\text { Luciana Araújo dos Reis; Stênio Fernando } \\
\text { Pimentel Duarte }\end{array}$ & $\begin{array}{l}\text { Conhecimento de enfermeiras em Uni- } \\
\text { dades de Saúde sobre a assistência à } \\
\text { mulher vítima da violência }\end{array}$ & Rev. Baiana enferm (2014) \\
\hline
\end{tabular}

Tabela 2- Descrição dos objetivos, instrumento utilizado e síntese dos resultados e conclusões dos estudos incluídos na análise:

\begin{tabular}{|c|l|l|l|}
\hline 01 & \multicolumn{1}{|c|}{ Objetivo } & \multicolumn{1}{c|}{ Análise } & \multicolumn{1}{c|}{ Síntese dos Resultados } \\
\hline $\begin{array}{l}\text { Entender a percepção } \\
\text { e abordagem de enfer- } \\
\text { meiros da Estratégia } \\
\text { (ESF) à mulher vítima } \\
\text { de violência }\end{array}$ & $\begin{array}{l}\text { Estudo qualitativo, } \\
\text { realizado com 13 } \\
\text { enfermeiros que atuam } \\
\text { na ESF de um municí- } \\
\text { pio de Mato Grosso } \\
\text { do Sul }\end{array}$ & $\begin{array}{l}\text { Os enfermeiros obtêm sentimentos de frustações ao atenderem } \\
\text { mulheres vítimas de violência, devido ao desfecho final da situ- } \\
\text { ação, pois geralmente as mulheres retornam ao ambiente junto ao } \\
\text { agressor. } \\
\text { É necessário criar vínculo com as vítimas e os agentes comuni- } \\
\text { tários em saúde são responsáveis por auxiliarem neste processo. } \\
\text { As consultas de enfermagem, especialmente as do pré-natal, são } \\
\text { fontes para identificar a violência. } \\
\text { Quando há percepção da possibilidade da violência, o enfermeiro } \\
\text { foca na conversa e vínculo, para que a vítima aceite ajuda }\end{array}$ \\
\hline $\begin{array}{l}\text { Analisar, baseado na } \\
\text { Teoria de Enfermagem } \\
\text { de Levine, o atendi- } \\
\text { mento da enfermeira } \\
\text { às mulheres que sofre- } \\
\text { ram violência }\end{array}$ & $\begin{array}{l}\text { Estudo qualitativo e } \\
\text { descritivo realizado na } \\
\text { Estratégia de Saúde } \\
\text { da Família (PSF) do } \\
\text { Rio de Janeiro - Brasil, } \\
\text { com 11 enfermeiras } \\
\text { que atenderam mul- } \\
\text { heres em situação de } \\
\text { violência }\end{array}$ & $\begin{array}{l}\text { Enfermeiras que prestam atendimento às mulheres vítimas de } \\
\text { violência buscam ofertar ferramentas que potencializem a inde- } \\
\text { pendência das mulheres (dar força a ela para continuar cuidando } \\
\text { dos filhos, prestação de cuidado integral, priorizar a cura dos } \\
\text { hematomas, tratar DSD) preservando sua saúde biopsicossocial e } \\
\text { tendo uma visão holística da vítima. } \\
\text { O enfermeiro sente-se inseguro diante de algumas situações, } \\
\text { procuram apoio das redes e da equipe multidisciplinar que é es- } \\
\text { sencial nesse processo. }\end{array}$ \\
\hline
\end{tabular}




\begin{tabular}{|c|c|c|c|}
\hline 03 & $\begin{array}{l}\text { Compreender as } \\
\text { motivações da ação } \\
\text { das enfermeiras no } \\
\text { cuidado de mulheres } \\
\text { vítimas de violência. }\end{array}$ & $\begin{array}{l}\text { Estudo qualitativo com } \\
\text { participação de } 10 \\
\text { profissionais enfermei- } \\
\text { ros }\end{array}$ & $\begin{array}{l}\text { A intencionalidade da ação desvelou a busca inicial da recuper- } \\
\text { ação da saúde física das mulheres, permeada pela expectativa } \\
\text { de compreender a situação; proporcionar bem-estar emocional, } \\
\text { apoio e a continuidade do cuidado, para que as mulheres possam } \\
\text { construir uma vida sem violência. } \\
\text { Demonstrou a importância de enxergar a integralidade de cada } \\
\text { mulher atendida, respeitando sua individualidade, mostrando a } \\
\text { elas as ferramentas e redes de apoio para minimizar ou deixar de } \\
\text { viver em situação de violência. } \\
\text { Evidencia a necessidade de aprimoramento dos profissionais no } \\
\text { que se refere ao acolhimento e escuta qualificada para um atendi- } \\
\text { mento efetivo. }\end{array}$ \\
\hline 04 & $\begin{array}{l}\text { Averiguar a atuação } \\
\text { dos enfermeiros refer- } \\
\text { ente à violência sexual } \\
\text { contra mulheres. }\end{array}$ & $\begin{array}{l}\text { Estudo quantitativo, } \\
\text { com análise de } 27 \\
\text { enfermeiros da rede } \\
\text { municipal de saúde do } \\
\text { município de Campina } \\
\text { Grande, PB, Brasil }\end{array}$ & $\begin{array}{l}\text { O estudo identificou que } 77 \% \text { dos enfermeiros não questiona } \\
\text { suas pacientes em relação à violência doméstica/sexual; } 85,1 \\
\% \text { já atenderam casos suspeitos e/ou confirmados de violência } \\
\text { sexual; } 88,8 \% \text { não utilizou algum protocolo durante o atendimento } \\
\text { e } 62,9 \% \text { fez algum encaminhamento para vítima. } \\
\text { Referente a avaliação quanto ao preparo das UBS quanto ao } \\
\text { atendimento a vítimas de violência sexual feminina, } 63 \% \text { dos } \\
\text { profissionais acreditam que a Unidade não está apta para atender } \\
\text { casos de violência sexual; } 77,8 \% \text { refere que não há um protocolo } \\
\text { para atendimento de violência sexual; } 63 \% \text { das unidades possuem } \\
\text { ficha de notificação compulsória para casos de violência contra a } \\
\text { mulher e } 100,0 \% \text { não teve em seis meses nenhum treinamento/ } \\
\text { capacitação sobre violência sexual contra a mulher. } \\
\text { Referente as dificuldades e sugestões encontradas pelos enfer- } \\
\text { meiros em relação ao atendimento a mulheres vítimas de violência } \\
\text { sexual, } 66,7 \% \text { não sente-se preparado profissionalmente para at- } \\
\text { ender casos de violência sexual; } 30 \% \text { possui maior dificuldade nos } \\
\text { aspectos éticos e legais; } 29,3 \% \text { referem ausência de treinamento } \\
\text { em serviço para atendimento a estas vítimas e } 30 \% \text { menciona que } \\
\text { as Oficinas de trabalho seriam a forma de treinamento mais eficaz } \\
\text { para obtenção de informação e capacitação dos enfermeiros } \\
\text { acerca da violência sexual. }\end{array}$ \\
\hline 05 & $\begin{array}{l}\text { Descrever a assistên- } \\
\text { cia à mulher vítima da } \\
\text { violência em unidades } \\
\text { de saúde no município } \\
\text { de Vitória da Conquista } \\
\text { (BA) e na contextual- } \\
\text { ização de trabalho das } \\
\text { coordenadoras dessas } \\
\text { unidades. }\end{array}$ & $\begin{array}{l}\text { Estudo exploratório } \\
\text { e descritivo, com } \\
\text { pesquisa de campo } \\
\text { e abordagem quanti- } \\
\text { tativa. Realizado no } \\
\text { município de Vitória da } \\
\text { Conquista (BA) com } 20 \\
\text { enfermeiras coordena- } \\
\text { doras de Unidades de } \\
\text { Saúde. }\end{array}$ & $\begin{array}{l}\text { A maioria dos profissionais enfermeiros ( } 75 \%) \text { demostraram con- } \\
\text { hecer a política de proteção à mulher e } 60 \% \text { já identificou casos } \\
\text { de violência na USF que trabalha. } 70 \% \text { julgam necessário haver } \\
\text { integração da violência contra a mulher no planejamento e ações } \\
\text { de assistência da unidade, com } 30 \% \text { julgando necessário cursos } \\
\text { de capacitação e atualização em violência contra a mulher na } \\
\text { unidade anualmente ou as vezes. } 85 \% \text { das enfermeiras relatam } \\
\text { mudanças na assistência à mulher vítima de violência após a } \\
\text { realização dos cursos e } 70 \% \text { referem haver dificuldades da equipe } \\
\text { para lidar com a violência contra a mulher. }\end{array}$ \\
\hline
\end{tabular}

\section{DISCUSSÃO}

Os achados sugerem que a temática "violência contra a mulher" vem sendo estudada no contexto brasileiro, sendo de suma importância aos enfermeiros, que estão na linha de frente dos atendimentos em saúde.

Juntamente há a expansão com novas reformulações políticas para a temática, como a recente institucionalização do decreto $\mathrm{N}^{\circ} 9.586$, de 27 de novembro de 2018, que tem por objetivo ampliar e fortalecer a formulação e a execução de políticas públicas de direitos das mulheres, de enfrentamento a todos os tipos de violência e da inclusão das mulheres nos processos de desenvolvimento social, econômico, político e cultural do país ${ }^{13}$.

Os estudos incluídos evidenciaram que os enfermeiros buscam ofertar atendimento integral às mulheres vítimas de violência, buscando ferramentas para potencializar a independência das mulheres e preservar sua saúde biopsicossocial por meio de uma visão holística da vítima. Durante os atendimentos, os enfermeiros referem que as mulheres vítimas de violência possuem dificuldade em relatar o problema. Deste modo, estabelecer vínculo e conquistar a confiança da paciente torna-se 
necessário para o atendimento integral, fazendo deste modo o vínculo com o paciente tornar-se essencial ${ }^{8,9}$

Os enfermeiros acreditam que as consultas de enfermagem, especialmente as de pré-natal, possam ser veículo de grande importância para criação de vínculo entre profissional-paciente e identificar a violência contra a mulher. Os estudos também referem a importância do trabalho da equipe multiprofissional no contexto da violência contra a mulher, pois os agentes comunitários são de extrema necessidade para trabalhar em parceria e obter informações para o atendimento integral e humanizado desta mulher, caracterizando a importância do trabalho da equipe multidisciplinar essencial nesse processo ${ }^{8,9}$.

A integralidade no atendimento de cada mulher atendida é necessária para respeitar sua individualidade, mostrando a elas as ferramentas e redes de apoio para minimizar ou deixar de viver em situação de violência ${ }^{10}$.

Apesar da oferta de cuidado integral e apoio a vítima, nota-se que os enfermeiros obtêm sentimentos de frustrações ao atenderem mulheres vítimas de violência, devido ao desfecho final da situação, pois geralmente as mulheres retornam ao ambiente junto ao agressor ${ }^{8,10}$. Contudo, os enfermeiros entendem seu papel como agente transformador, conscientizando as vítimas sobre a situação e oferecendo alternativas para mudança dessa realidade ${ }^{11}$.

Os estudos evidenciaram que grande parte dos enfermeiros não questiona suas pacientes em relação à violência doméstica/sexual e não utilizou nenhum protocolo durante o atendimento ${ }^{11}$. Deste modo, os achados evidenciam fortemente a necessidade de maiores investimentos nas capacitações de estudantes e profissionais da saúde atuantes nos serviços de saúde, para que estejam preparados para identificar e lidar com essa realidade e por meio do seu atendimento possa prestar atendimento seguro e resolutivo às vítimas, pois quase metade dos enfermeiros entrevistados em um estudo selecionado para análise, referem não conhecer as Leis e Decretos que tangem a legislação neste tema ${ }^{12}$.

\section{CONSIDERAÇÕES FINAIS}

A presente revisão integrativa da literatura identificou que os enfermeiros no contexto da violência contra a mulher buscam prestar atendimento integral às vítimas, para que haja recuperação da saúde física, permeada pela expectativa de compreender a situação buscando proporcionar bem-estar emocional, apoio e a continuidade do cuidado, para que as mulheres possam construir uma vida sem violência.

Estabelecer vínculo com as pacientes torna-se necessário para o cuidado, bem como o trabalho multiprofissional neste contexto. Além disso, existe a notória necessidade de aprimoramento dos profissionais no que se refere ao acolhimento e escuta qualificada para um atendimento efetivo. Os profissionais devem obter preparo desde a sua formação, para lidar com situações como essa e para estar apto a oferecer alternativas de mudança dessa realidade.

A necessidade de treinamento das equipes, bem como obtenção do conhecimento legislativo que assegura mulheres em situação de violência, deve ser de amplo conhecimento da equipe, para que a partir desse conhecimento desenvolvam-se ações de prevenção e proteção às vítimas, pois a ausência desse conhecimento pode ser fator limitante na atuação dos profissionais que lidam com essa realidade. 


\section{REFERÊNCIAS}

1. Albuquerque NL; Moura MAV; Queiroz ABA; Tyrrell, MAR; Bravo MPBrM. Violência contra a mulher e suas consequências. Acta Paul Enferm. 2014. [Acesso em 2019 mar 15]; 27(5):458-64. Disponível em: http://www.scielo.br/pdf/ape/v27n5/pt_1982-0194ape-027-005-0458.pdf

2. Cidreira MCCB. Cicatrizes da dor: as consequências da violência doméstica na saúde física da mulher. Trabalho apresentado na VII Jornada Internacional Políticas Públicas; 2014 [capturado 23 maio 2017]; São Luiz (MA). [Acesso em 2019 mar 15]; Disponível em:

http://www.joinpp.ufma.br/jornadas/joinpp2017/pdfs/ eixo6/cicatrizesdadorasconsequenciasdaviolenciadomesticanasaudefisicadamulher.pdf

3. BRASIL. Lei n 11.340 de 7 de agosto de 2006. Cria mecanismos para coibir a violência doméstica e familiar contra a mulher. Diário Oficial da União, Brasília (DF), 2006(ago.)08;Sec.1:56.

4. Porto PRF. Violência doméstica e familiar contra a muIher: Lei 11.340/06 análise crítica e sistêmica. Livraria do advogado Editora, 2018.

5. Porto RTS, Bispo Junior JP, Lima EC. Violência doméstica e sexual no âmbito da Estratégia de Saúde da Família: atuação profissional e barreiras para o enfrentamento. Physis [online]. 2014;24(3):787-807.

6. Paiva AC, Santos VRP, Santos SM. Violência doméstica e as implicações na saúde física emocional de mulheres: Inferências de Enfermagem. Trabalho apresentado no XI Simpósio de excelência em gestão e tecnologia; 2014; Resende (RJ). Disponível em:

https://www. aedb.br/seget/arquivos/artigos 14/23020192.pdf
7. Mendes KDS, Silveira RCCP, Galvão CM. Revisão integrativa: método de pesquisa para a incorporação de evidências na saúde e na enfermagem. Texto Cont Enferm. 2008;17(4):758-64.

8. Morais BLA, Gerk MAS, Nunes CB. Enfermeira da Estratégia de Saúde da Família: abordagem frente à mulher em situação de violência. Nursing (São Paulo). 2018;21(240):2164-2167.

9. Albuquerque NL, Pereira ER, Tavares JMAB, Ferreira DC. Atuação da enfermagem na conservação da saúde de mulheres em situação de violência. REME rev. min. Enferm. 2018;22: e-1149.

10. Cortes LF, Padoin SMM. Intencionalidade da ação de cuidar mulheres em situação de violência: contribuições para a Enfermagem e Saúde. Esc. Anna Nery Rev. Enferm. 2016;20(4):e20160083.

11. Baptista RS, Chaves OBBM, França ISX, Sousa FS, Oliveira MG, Leite CCS. Violência sexual contra mulheres: a prática de enfermeiros Rev. RENE. 2015;16(2):210-217.

12. Santos J, Andrade RL, Reis LA, Duarte SFP. Conhecimento de enfermeiras em unidades de saúde sobre a assistência à mulher vítima da violência. Rev. baiana enferm; 2014;28(3).

13. Brasil. Decreto n 9.586, de 27 de novembro de 2018. Institui o Sistema Nacional de Políticas para as Mulheres e o Plano Nacional de Combate à Violência Doméstica. [Acesso em 2020 maio 27]; Disponível em: http://www. planalto.gov.br/CCIVIL_03/_Ato2015-2018/2018/Decreto/D9586.htm. 